\title{
CARACTERIZAÇÃO FUZZYDO TRANSPORTE PRÉ-ABATE DE FRANGOS DE CORTE: UMA ABORDAGEM QUALITATIVA
}

\author{
FUZZY CHARACTERIZATION OF PRESLAUGHTERBROILERTRANSPORT: \\ AQUALITATIVEAPPROACH \\ Rodrigues-Sarnighausen, V.C. ${ }^{1}$; Vieira, F.M.C. ${ }^{1}$; Silva, I.J.O. ${ }^{1}$; Barbosa Filho, J.A.D. ${ }^{2}$ \\ e Vieira, A.M.C. ${ }^{3}$
}

\begin{abstract}
1'Departamento de Engenharia de Biossistemas. ESALQ-USP.Piracicaba-SP.Brazil. vcrodrig@esalq.usp.br ${ }^{2}$ Departamento de Engenharia Agrícola. Universidade Federal do Ceará (UFC). Fortaleza. Ceará. Brazil. ${ }^{3}$ Departamento de Estatística. Universidade de Brasília. Brasília. Distrito Federal. Brazil.
\end{abstract}

\section{PALAVRAS CHAVE ADICIONAIS \\ Graus de pertinência. Planejamento.}

\section{RESUMO}

A produção de carne de frango no Brasil é um dos setores mais importantes do agronegócio, sendo que a expansão e manutenção dos mercados externos estão diretamente relacionadas com a qualidade do produto final. Um dos grandes problemas no sistema de produção de frangos, esta relacionado com a logística de distribuição e conseqüentemente as formas de transporte de cargas vivas. Nesta pesquisa objetivou-se determinar a classificação do nível de perdas produtivas na carga viva de frangos de corte, utilizando como ferramenta de decisão a lógica fuzzy. Após a avaliação das informações de entrada no sistema fuzzy verificou-se que o modelo obtido é importante por dois fatores: o primeiro pela classificação clara e direta das condições de transporte e segundo pela perspectiva de estabilidade ou não das condições classificadas, mostrando o potencial de mudança das mesmas, o que poderá ser utilizada como objeto de atenção para a escolha de estratégias de transporte.

\section{SUMMARY}

The poultry industry at Brazil is one of the most important sectors of agribusiness, which expansion and maintenance of the international markets are directly linked with the final product quality. One of the major problems on the poultry production system is related with distribution logistics and consequently with the types of

\author{
AdDITIONAL KEYWORDS \\ Pertinence degrees. Planning.
}

livestock transport. In this research, the objective was to determine the classification of productive losses levels on the poultry transport, using as decision tool the fuzzy logic. After the assessment of input information on fuzzy system, it was verified that the developed model is important due two reasons: firstly, to the clear and direct classification of transport conditions, and secondly to the perspective of the stability or not of the assorted condition, showing this potential for change, which may be used with the aim to pay attention during the choose of transport strategies.

\section{INTRODUÇÃO}

A preocupação com o bem estar de frangos de corte nas operações pré-abate é crescente, com destaque em regiões tropicais. Apesar de outros fatores influírem nas perdas produtivas, como as injúrias e traumas provenientes da pega e do carregamento, distância entre as granjas e o abatedouro, vibração do caminhão e restrição de água, os fatores térmicos correspondem cerca de $40 \%$ das perdas totais (Ritz et al., 2005).

No transporte, há uma variação térmica por volta de 2 a $5^{\circ} \mathrm{C}$ entre o microclima interno das caixas e o ambiente externo (Mitchell e Kettlewell, 1998). Esses fatores, 
como a elevada temperatura e umidade provenientes das aves (calor latente e sensível), heterogeneidade provocada pela distribuição da ventilação, resultam em mortalidade, também chamadas de mortes na chegada (Hunter et al., 1997).

Neste cenário o uso da lógica fuzzy é uma forma de investigar, em conformidade com os conhecimentos de especialistas, possíveis termos lingüísticos que atribuam valores à relação entalpia-período de transporte, gerando informações de suporte a decisão. Assim, objetivou-se por meio da lógica fuzzy determinar um modelo para classificar e predizer o nível de perdas produtivas relacionadas ás variáveis climáticas da carga viva durante o transporte de frangos de corte.

\section{MATERIALEMÉTODOS}

A pesquisa foi realizada nos meses de abril a agosto de 2006 (inverno), e de dezembro a março de 2007 (verão), em caminhões de transporte de frangos de corte em condições tropicais. Foram monitorados 16 carregamentos no total, 8 carregamentos no inverno e 8 carregamentos no verão. As cargas dos caminhões totalizavam, em média, 486 caixas, contendo de 6 a 9 aves por caixa. Os períodos de transporte considerados foram: manhã (6:00 às $12: 00$ horas), tarde (12:00 às 18:00 horas) e noite (18:00 às 00:00 horas). As aves utilizadas foram da linhagem Cobb, com idades variando de 6 a 7 semanas.

As variáveis ambientais (temperatura e umidade relativa) foram monitoradas ao longo de toda a carroceria do caminhão, por meio da instalação de data loger em várias alturas e distâncias ao longo da carga, formando uma malha de pontos, conforme evidenciado em Barbosa Filho et al. (2009).

Os valores da temperatura $\left(t_{D b},{ }^{\circ} \mathrm{C}\right) \mathrm{e}$ umidade relativa do ar ( $\mathrm{RH}, \%)$ foram utilizados para o cálculo da entalpia específica da carga $(h, \mathrm{~kJ} / \mathrm{kg}$ de ar seco), adotada por Barbosa Filho et al. (2006). Valores de entalpia e horários condizentes aos períodos de transporte foram utilizados para a elaboração de um perfil qualitativo para classificar a operação de transporte (bom, regular e inapropriado), baseando-se nos valores discretos das taxas de mortalidade.

Para esta avaliação, foi utilizado a lógica fuzzy, por meio de informações que definem tipos de adesão de um valor a determinadas classes, sem a obrigatoriedade dos mesmos pertencerem $100 \%$ a uma classe qualquer (Zadeh, 1965). Para cada variável (períodos e entalpia) foram atribuídas faixas, ou classes, que condizem com determinada situação. Para a entalpia, existem 4 faixas que retratam as situações de conforto, crítica, alerta e letal para frangos de corte na sexta semana. Foi utilizada para este trabalho, a função sino-generalizada. As fronteiras são bem delimitadas criando mudanças suaves de uma classe para outra, trata-se de uma extensão da função de distribuição de probabilidade de Cauchy, dependente de 3 parâmetros (Vaz, 2006).

Considerando que $x$ é a variável entalpia que pode estar relacionada às 4 classes lingüísticas $A_{j}\left(A_{1}\right.$ - conforto, $A_{2}-$ crítico, $A_{3}$ - alerta e $A_{4}$-letal). E yé a variável relacionada aos períodos, ou seja, a variável lingüística $B_{k}\left(B_{1}-\right.$ manhã, $B_{2}-$ tarde, $B_{3}-$ noite $)$. A variável resposta $z$ é a qualidade atribuída ao transporte que foi orientada no intervalo numérico de [0 1], codificando três faixas de qualidade (variável lingüística): bom, regular e inapropriado.

O sistema fuzzy utilizado apresentou os seguintes elementos:

1. Entradas: entalpia e período;

2. Fuzzificadores: transformar dados numéricos em faixas de valores;

3. Regras fuzzy: inferências, funções de pertinências, correspondência entre entradas e sáidas;

4. Defuzzificadores: transformação da saída fuzzy em dados numéricos.

Para a obtenção das funções de pertinência, fez-se necessária a apresentação dos intervalos fuzzy (Weber e Klein, 2003), 


\section{CARACTERIZAÇÃO FUZZYDE TRANSPORTE DE FRANGOS DE CORTE}

os quais mostram que valores de fronteira como entalpia de $75,8 \mathrm{~kJ} / \mathrm{kg}$ de ar seco, pertencem a duas classes (alerta e letal) de forma que a função de pertinência atribui $50 \%$ de correspondência com cada faixa.

A faixa de mortalidade foi de 0,10 a $0,23 \%$ no inverno e de 0,12 a $0,33 \%$ no verão. Foram adotadas faixas de qualidade de transporte num intervalo de [0 1]. Esta faixa foi normalizada e divida em proporções iguais em três faixas do sistema fuzzy, caracterizando os termos bom, regular e inapropriado. A faixa de mortalidade, englobando inverno e verão apresentou valores entre 0,10 e $0,33 \%$ de perdas. Estes foram normalizados de forma simétrica para compor valores tais de 0,10 a $0,18 \%$ a valores lingüísticos bom para o transporte, com os maiores índices de qualidade (entre 0,55 a 1). A situação regular foi caracterizada como mortalidade entre 0,18 a $0,25 \%$ e índices de qualidade entre 0,25 e 0,75 . Para o transporte inapropriado a faixa de mortalidade foi a maior $(0,25$ a $0,33 \%)$ e valores menores de índica de qualidade $(0$ a 0,45$)$. Como pode ser visto, há uma convergência de valores entre os índices, o que faz com que o sistema de classificação fuzzy possa medir o quanto regular, por exemplo, é um transporte, se há a possibilidade de ajustar mais próximo de um bom índice ou se há tendência em ser caracterizado por inapropriado.

Após obtenção dos conjuntos fuzzy e regras com os termos lingüísticos, a adoção de um sistema de defuzzificação foi necessária para se obter um valor numérico associado às regras anteriormente estipuladas, de forma a caracterizar a qualidade do transporte relacionada às perdas na chegada (DOA). Para estas etapas, foi utilizado o método Mamdani para a caracterização das regras (Mamdani, 1975). O método de defuzzificação adotado foi o centróide, mais comumente utilizado. É referenciado como método do centro de gravidade. O software Matlab®2006a foi utilizado para implementação das regras por meio do toolbox de lógica fuzzy.

\section{RESULTADOSEDISCUSSÃO}

Os valores de entalpia $(h)$ entre 40 a 54,7 $\mathrm{kJ} / \mathrm{kg}$ de ar seco são da época de inverno e apresentaram duas caracterizações com termos lingüísticos semelhantes às faixas de verão $(54,7$ a $90,8 \mathrm{~kJ} / \mathrm{kg}$ de ar seco). Os termos de inverno são condizentes a situações de estresse por frio e demais faixas são relacionadas ao estresse por calor. As faixas de entalpia são características das condições do ambiente térmico da carroceria do caminhão, porém, não é o suficiente para informar o nível de estresse térmico ao quais os animais ficam submetidos. A partir do conjunto de 18 regras relacionaram-se informações e definiu-se um panorama das condições do transporte baseado em dados de mortalidade analisados por especialistas. Na figura 1 observa-se a superfície de resposta relacionada com a qualidade do transporte (índice de 0 a 1 ) em função do período e entalpia. É um gráfico em terceira dimensão cujo eixo principal (y) mostra a qualidade do transporte, entre 0 e 1 conforme a codificação do sistema fuzzy.

Nota-se que o período da manhã apresentou índice de qualidade de trans-

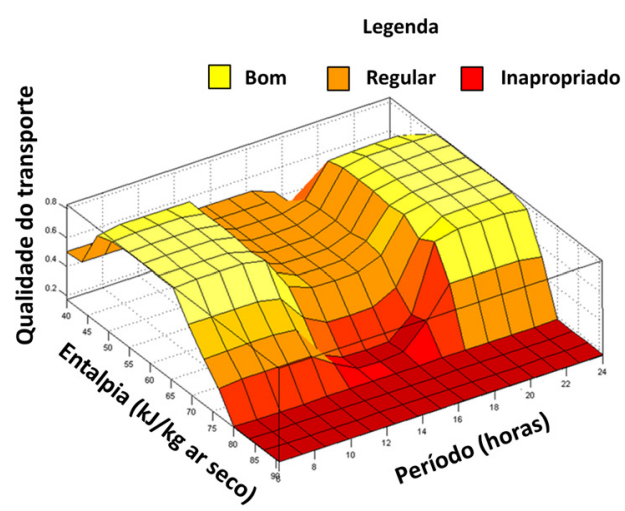

Figura 1. Superfície resposta de regras considerando período de transporte, entalpia e qualidade do transporte. (Response surface of rules considering transport period, enthalpy and transport quality). 


\section{RODRIGUES, VIEIRA, SILVA, BARBOSA FILHOE VIEIRA}

porte entre 0 a 0,8 ; o que indica que houve situações características de ambiente bom ao inapropriado. Neste período, entre a faixa de entalpia de 45 a $60 \mathrm{~kJ} / \mathrm{kg}$, a classificação foi bom, dentro do esperado conforme os conjuntos de regra de referência, além disso, é evidente o decréscimo da qualidade de transporte com o aumento da entalpia, característico de situações de verão.

A faixa de 40 a $45 \mathrm{~kJ} / \mathrm{kg}$ de ar seco mostra a faixa de situação regular, devido ao frio favorecido pela baixa entalpia e período do dia. De acordo com Bayliss e Hinton (1990), há um aumento significativo de mortalidade no período da manhã no inverno, explicado pela variação dessa faixa na figura, e as taxas de mortalidade também aumentaram no verão, no período da tarde.

A qualidade do transporte foi indicada pelos autores como relações diretas relacionadas às taxas de mortalidade durante experimentação. Isto propiciou a classificação das condições com vista nas possí-

\section{BIBLIOGRAFIA}

Barbosa Filho, J.A.D.; Silva, M.A.N.; Vieira, F.M.C. e Silva, I.J.O. 2006. Avaliação direta e prática caracterização do ambiente interno de galpões de criação de frangos de corte utilizando tabelas práticas de entalpia. Avicultura Industrial, 1144: 54-57.

Barbosa Filho, J.A.D.; Vieira, F.M.C.; Silva, I.J.O.; Garcia, D.B.; Silva, M.A.N. e Fonseca, B.H.F. 2009. Transporte de frangos: caracterização do microclima na carga durante o inverno. Rev Bras Zootecn, 38: 2442-2446.

Bayliss, P.A. and Hinton, M.H. 1990. Transportation of broilers with special reference to mortality rates. Appl Anim Behav Sci, 28: 93-118.

Hunter, R.R.; Mitchell, M.A. and Matheu, C. 1997. Distribution of 'dead on arrivals' within the bioload on commercial broiler transporters: correlation with climate conditions and ventilation regimen. Brit Poultry Sci, 38: 7-9.

Mamdani, E.H. and Assilan, S.A. 1975. An expe- veis mudanças. Uma classificação boa de menor índice de qualidade indica o potencial de modificação para uma situação $r e g u$ lar. Portanto o modelo é válido para ser utilizado num sistema de informação e decisão pautadas em dados reais. Com base em informações lingüísticas, é possível reconhecer as condições em que a carga é transportada e seu potencial de melhorias de forma simples e direta.

\section{CONCLUSÕES}

O modelo fuzzy indica a possibilidade de classificação das condições de transporte e propicia um panorama de potencialidades de mudanças. É possível além de classificar, considerar as possíveis mudanças que esta dada classificação pode apresentar num processo, ou seja, se a classificação é estável ou não e ainda quais as tendências de mudanças. Este modelo poderá ser utilizado para compor um sistema de informação e tomadas de decisão em tempo real.

riment in linguistic synthesis with a fuzzy logic controller. Int J Man-Machine Studies, 7: 1-13.

Mitchell, M.A. and Kettlewell, P.J. 1998. Physiological stress and welfare of broiler chickens in transit: Solutions not problems. Poultry Sci, 77: 1803-1814.

Ritz, C.W.; Webster, A.B. and Czarick, M. 2005. Evaluation of hot weather thermal environment and incidence of mortality associated with broiler live haul. J Appl Poultry Res, 14: 594-602.

Vaz, A.M. 2006. Estudos das funções de pertinência para conjuntos fuzzy utilizados em controladores semafóricos fuzzy. Dissertação (Mestrado em Engenharia Civil e Ambiental). Faculdade de Tecnologia. Universidade de Brasília. Brasília.

Weber, L. e Klein, P. 2003. Aplicação da lógica fuzzy em software e hardware. Ulbra. 175 pp.

Zadeh, L.A. 1965. Fuzzy Sets. Information and control, 8: 330-353. 